\title{
Fine-tuning immunotherapy in MMR-D/MSI-H colorectal cancer
}

\author{
Ibrahim Halil Sahin*,1 (iD \\ ${ }^{1}$ Department of Hematology/Oncology, Emory University School of Medicine, Winship Cancer Institute, GA 30322, USA \\ *Author for correspondence: insahin@emory.edu
}
" based on the durable response with immune checkpoint inhibitors, BRAF mutant MMR-D/MSI-H colorectal patients should be considered for immunotherapy over BRAF-targeted agents"

First draft submitted: 18 November 2019; Accepted for publication: 22 November 2019; Published online: 9 January 2020

Keywords: colorectal cancer $\bullet$ immune checkpoint inhibitor $\bullet$ immunotherapy • ipilimumab • MLH-1 • MMR-D • MSH-2 • MSH-6 • MSI-H • nivolumab • pembrolizumab • PMS-2

Colorectal cancer with mismatch repair deficiency (MMR-D) has been a focus of interest for cancer researchers due to its unique features, including increased mutation-associated neoantigen (MANA) generation and the presence of tumor-infiltrating lymphocytes in the tumor microenvironment. MMR-D, defined as the loss of function in any of four MMR genes (MLH1, PMS2, MSH2 and MSH6), sets off defective DNA repair - particularly during the DNA replication process - leading to microsatellite instability (MSI), which causes frameshift mutations in the DNA [1]. Based on the number or percentage of altered microsatellite markers, MSI can be classified into MSI-low (MSI-L) and MSI-high (MSI-H) [2]. Unlike point mutations, frameshift mutations frequently result in significant changes in the amino acid sequence of affected proteins. This change forms MANAs leading to the induction of adaptive immune system elements, particularly cytotoxic $T$ cells [3]. This immune response against MANA constitutes the basis for the recent therapeutic success of immune checkpoint inhibitors in MMR-D/MSI-H colorectal cancer patients.

Pembrolizumab, an anti-PD-1 antibody, became the first immune checkpoint inhibitor approved in MMRD/MSI-H cancer patients based on an objective response rate (ORR) of 40\%, which was durable [4]. Notably, this benefit was observed across all MMR-D/MSI-H cancers and thus, regardless of the tumor histology, the US FDA granted approval to pembrolizumab as a second line or subsequent therapy [5]. In this study, the authors identified that the MANAs may be the surrogate for the duration of response, suggesting that the major determinant of the immune response is the quality/nature of the mutation(s), rather than their quantity. This study was followed by clinical trials investigating the efficacy of nivolumab (an anti-PD1 antibody) and nivolumab, in combination with ipilimumab (a cytotoxic T lymphocyte-associated protein-4 [CTLA4] antibody). Both studies revealed promising results with ORR ranging between 31 and 55\%, in metastatic MMR-D/MSI-H colorectal cancer patients who were previously treated with at least one line of chemotherapy [6,7]. Notably, in these studies, the authors investigated biomarkers of immune checkpoint inhibitor response including, $P D-1$ expression and $B R A F / K R A S$ mutations. Curiously, none of these markers correlated with clinical outcomes. However, in the study of nivolumab and ipilimumab combination [7], the ORR was $71 \%$ in patients with a history of Lynch syndrome, which was numerically higher than when compared with patients who had sporadic MMR-D/MSI-H disease (48\%). This notable finding suggests that there may be disease heterogeneity among MMR-D/MSI-H colorectal cancer patients, particularly with $B R A F$-driven MMR-D/MSI-H colorectal cancer, which frequently occurs as a result of hypermethylation of the $M L H 1$ promoter region. However, the durability of response was not examined by $B R A F$ mutation and Lynch syndrome status, which are fundamental causes of disease heterogeneity among MMR-D/MSI-H colorectal cancer patients.

It is also important to note, MMR-D-associated colorectal cancer may present as MSI-L disease, which carries a relatively low mutation burden when compared with MSI-H tumors. It is unclear at this time if this leads to a distinct clinical course in patients treated with immune checkpoint inhibitors [2]. Notably, immunohistochemistry,

Future $\because$ Medicine 
which is a commonly used method in clinical practice, can only detect MMR-D status by identifying the loss of expression-specific MMR genes, whereas MSI status can be identified either by performing PCR or nextgeneration sequencing. Currently, the field is moving toward next-generation sequencing-based molecular profiling platforms, which can precisely differentiate MSI-H from MSI-L and can concurrently identify specific MMR gene(s) mutation(s) [8].

Although immune checkpoint inhibitors have led to significant shifts in clinical approaches to MMR-D/MSI$\mathrm{H}$ colorectal cancer, upfront or subsequent failure of these agents have been reported [4,7], suggesting de novo (intrinsic) and acquired resistance mechanisms to these agents. At this time, the exact mechanisms of resistance remain unclear. However, accumulating evidence suggests that there may be multiple distinct pathways that are associated with immune evasion and underly the failure of immune checkpoint inhibitors in solid tumors, even those that are highly immunogenic, for example, MMR-D/MSI-H colorectal cancer [9]. Recent studies revealed frequent alterations in antigen presentation pathways, which impair recognition of MANAs by effector T cells [10]. That includes biallelic loss of $\beta 2$-microglobulin - an important element of the MHC-I antigen-presenting machinery, antigen peptide transporters (TAP1/2) as well as the loss of MHC-I expression on the cell surface [10]. Notably, the $\beta 2$-microglobulin gene has coding microsatellites, which also make this gene frequently mutated in MSI-H colorectal cancer patients. Molecular alterations leading to loss of function, particularly biallelic loss, in STAT and JAK2 genes which are important mediators of IFN- $\gamma$ signaling, have been identified as possible resistance mechanisms to immune checkpoint inhibitors [11]. Although these mechanisms may play roles in both de novo/intrinsic and acquired resistances, they appear to primarily cause early resistance in parental clones and subsequently lead to failure of immune checkpoint inhibitors.

The tumor microenvironment and pathways such as, Wnt and TGF- $\beta$ signaling, may also impair effective cytotoxic T-cell responses [10]. Recent evidence suggests that the negative regulatory role of Wnt and TGF- $\beta$ on effector T-cell function via the upregulation of $\mathrm{T}$ regulatory cells, may impair the effectiveness of immune checkpoint inhibitors [12]. Collectively, growing data indicate that effective anti-cancer responses can be altered by both intrinsic and extrinsic mechanisms, which may abolish the effectiveness of immune checkpoint inhibitor therapy in MMR-D/MSI-H patients.

So far, three immunotherapeutic choices have been granted approval by the FDA; these options include pembrolizumab, nivolumab and nivolumab, in combination with ipilimumab, in MMR-D/MSI colorectal cancer patients who were previously treated with cytotoxic therapies. At this time, there is no comparative data on the efficacy of these agents, particularly single-agent versus combination therapy. However, a study by Overman et al. [7] compared two cohorts; patients treated with single-agent nivolumab versus patients treated with nivolumab plus ipilimumab. The Kaplan-Meier survival curve of the combination therapy appears superior to single-agent nivolumab. Although the cohorts were not randomized and represent two different study populations with inherent disease heterogeneity, the 24-month disease control rate also favored the combination of anti-PD-1 and anti-CTLA4 antibodies for improved outcomes. It is important to note that the combination of anti-PD-1 and anti-CTL antibodies potentially results in higher immune-related adverse events [13] when compared with singleagent immune checkpoint inhibitors. Therefore, the clinical decision regarding the choice of single-agent versus combination therapy should be made on a case-by-case basis, depending on the clinical characteristics of each individual patient. Perhaps patients with multiple medical comorbidities and poor performance status or individuals who are at high risk for autoimmune disorders may not be good candidates for combination therapy. Patients with an active autoimmune disorder or a history of solid organ transplants should not be exposed to these agents, even single agent immune checkpoint inhibitors.

\section{Future perspective $\&$ conclusion}

Although these highly promising agents have changed the clinical practice in this subset of colorectal cancer patients, there is still significant room for improvement. At this time, there is no established second-line immune checkpoint inhibitor for use in patients who have previously treated with one. Therefore, these patients should be considered for clinical trials that are investigating novel checkpoint inhibitors with new targets such as anti-LAG3 (NCT03607890) or novel immunomodulatory agents, which may overcome immune exhaustion [14]. Moreover, potentially synergistic roles of Wnt and TGF- $\beta$ inhibitors, in combination with immune checkpoint inhibitors should be further investigated in MMR-D/MSI-H colorectal cancer patients [15]. Approaches with combinations of cytotoxic agents with immune checkpoint inhibitors have achieved substantial success in non-small-cell lung cancer [16]. Similarly, the role of chemoimmunotherapy in MMR-D/MSI-H colorectal cancer patients deserves 
further attention. Poly (ADP)-ribose polymerases (PARP) inhibitors, which are novel agents that are very effective in the setting of homologous recombination DNA repair defect (synthetic lethality), also demonstrated synergistic effects with immune checkpoint inhibitors in selected solid tumors such as triple-negative breast cancer [17]. Potential additive benefit of PARP inhibitors in MMR-D/MSI-H colorectal cancer patients also warrants further evaluation. Last, the biology of $B R A F$-driven MMR-D/MSI-H colorectal cancers appears to be diverse and future studies are required to better understand this heterogeneity among this unique subset of patients. Although recent studies revealed promising outcomes with $B R A F$-targeted therapies [18], based on the durable response with immune checkpoint inhibitors, $B R A F$ mutant MMR-D/MSI-H colorectal patients should be considered for immunotherapy over $B R A F$-targeted agents [19].

Overall, immune checkpoint inhibitors have led to significant changes in the horizons of cancer therapy in highly immunogenic tumors, including MMR-D/MSI-H colorectal cancer. In the near future, these agents will likely be moved up to first-line therapy for metastatic MMR-D/MSI-H colorectal cancer, given current observed durable responses. The future holds many opportunities to enhance therapeutic options with these agents using different combination approaches in various clinical settings, perhaps including adjuvant therapy. Therefore, future prospective clinical studies will hopefully move the field forward for more therapeutic options in MMR-D/MSI-H colorectal cancer patients.

\section{Financial \& competing interests disclosure}

The authors have no relevant affiliations or financial involvement with any organization or entity with a financial interest in or financial conflict with the subject matter or materials discussed in the manuscript. This includes employment, consultancies, honoraria, stock ownership or options, expert testimony, grants or patents received or pending, or royalties.

No writing assistance was utilized in the production of this manuscript.

\section{Ethical conduct of research}

The authors state that they have obtained appropriate institutional review board approval or have followed the principles outlined in the Declaration of Helsinki for all human or animal experimental investigations. In addition, for investigations involving human subjects, informed consent has been obtained from the participants involved.

\section{Open access}

This work is licensed under the Attribution-NonCommercial-NoDerivatives 4.0 Unported License. To view a copy of this license, visit http://creativecommons.org/licenses/by-nc-nd/4.0/

\section{References}

1. Eshleman JR, Markowitz SD. Mismatch repair defects in human carcinogenesis. Hum. Mol. Genet. 5(Supplement 1), 1489-1494 (1996).

2. Pawlik TM, Raut CP, Rodriguez-Bigas MA. Colorectal carcinogenesis: MSI-H versus MSI-L. Dis. Markers 20(4,5), 199-206 (2004).

3. Liu B, Nicolaides NC, Markowitz S et al. Mismatch repair gene defects in sporadic colorectal cancers with microsatellite instability. Nat. Genet. 9(1), 48 (1995).

4. Le DT, Uram JN, Wang H et al. PD-1 blockade in tumors with mismatch-repair deficiency. N. Engl. J. Med. 372(26), 2509-2520 (2015).

5. Sahin I, Askan G, Hu Z, O’Reilly E. Immunotherapy in pancreatic ductal adenocarcinoma: an emerging entity? Ann. Oncol. 28(12), 2950-2961 (2017).

6. Overman MJ, Mcdermott R, Leach JL et al. Nivolumab in patients with metastatic DNA mismatch repair-deficient or microsatellite instability-high colorectal cancer (CheckMate 142): an open-label, multicentre, Phase II study. Lancet Oncol. 18(9), 1182-1191 (2017).

7. Overman MJ, Lonardi S, Wong KYM et al. Durable clinical benefit with nivolumab plus ipilimumab in DNA mismatch repair-deficient/microsatellite instability-high metastatic colorectal cancer. J. Clin. Oncol. 36(8), 773-779 (2018).

8. Middha S, Zhang L, Nafa K et al. Reliable pan-cancer microsatellite instability assessment by using targeted next-generation sequencing data. JCO Precision Oncol. 1, 1-17 (2017).

9. Sahin IH, Akce M, Alese $\mathrm{O}$ et al. Immune checkpoint inhibitors for the treatment of MSI-H/MMR-D colorectal cancer and a perspective on resistance mechanisms. Br. J. Cancer 121, 809-818 (2019).

10. Grasso CS, Giannakis M, Wells DK et al. Genetic mechanisms of immune evasion in colorectal cancer. Cancer Discov. 8(6), 730-749 (2018).

11. Sveen A, Johannessen B, Tengs T et al. Multilevel genomics of colorectal cancers with microsatellite instability - clinical impact of JAK1 mutations and consensus molecular subtype 1. Genome Med. 9(1), 46 (2017). 
12. Spranger S, Bao R, Gajewski TF. Melanoma-intrinsic $\beta$-catenin signalling prevents anti-tumour immunity. Nature 523(7559), 231 (2015).

13. Larkin J, Chiarion-Sileni V, Gonzalez R et al. Combined nivolumab and ipilimumab or monotherapy in untreated melanoma. $N$. Engl. J. Med. 373(1), 23-34 (2015).

14. Sharma P, Allison JP. Immune checkpoint targeting in cancer therapy: toward combination strategies with curative potential. Cell 161(2), 205-214 (2015).

15. Guler I, Askan G, Klostergaard J, Sahin IH. Precision medicine for metastatic colorectal cancer: an evolving era. Expert Rev. Gastroenterol. Hepatol. 13, 919-931 (2019).

16. Gandhi L, Rodríguez-Abreu D, Gadgeel S et al. Pembrolizumab plus chemotherapy in metastatic non-small-cell lung cancer. N. Engl. J. Med. 378(22), 2078-2092 (2018).

17. Vinayak S, Tolaney SM, Schwartzberg LS et al. TOPACIO/Keynote-162: niraparib+ pembrolizumab in patients (pts) with metastatic triple-negative breast cancer (TNBC), a Phase II trial. 36, 1011 (2018).

18. Sahin IH, Klostergaard J. BRAF mutations as a therapeutic target in metastatic colorectal cancer patients: a long due success. 8, 1-5 (2019).

19. Kopetz S, Grothey A, Yaeger R et al. Encorafenib, binimetinib, and cetuximab in BRAF V600E-mutated colorectal cancer. N. Engl. J. Med. 381(17), 1632-1643 (2019). 